Check for updates

Cite this: Mater. Adv., 2021, 2, 2398

Received 9th February 2021, Accepted 21st February 2021

DOI: 10.1039/d1ma00122a

rsc.li/materials-advances

\title{
Anion ordering and vacancy defects in niobium perovskite oxynitrides $\dagger$
}

\author{
Joshua J. Brown, ${ }^{a}$ Youxiang Shao, ${ }^{b}$ Zhuofeng Ke $\mathbb{D}^{b}$ and Alister J. Page (D) *a
}

\begin{abstract}
Niobium perovskite oxynitrides are emerging as promising semiconductor materials for solar energy conversion processes, due to their physical properties and amenability to defect engineering. However, defect engineering in mixed-anion semiconductors such as perovskite oxynitrides is generally hindered by the absence of long-range order in the crystal lattice, a phenomenon known as anion-ordering. We demonstrate how anion ordering influences the stability and mobility of point defects in two exemplar perovskite oxynitrides, $\mathrm{BaNbO}_{2} \mathrm{~N}$ and $\mathrm{LaNbON}_{2}$. Accurate first-principles calculations show that fully cis-anion orderings in $\mathrm{BaNbO}_{2} \mathrm{~N}$ are more stable than fully trans-anion orderings, whereas anion orderings with mixed dimensionality may be more prevalent in the lower-symmetry $\mathrm{LaNbON}_{2}$. Anion ordering in $\mathrm{LaNbON}_{2}$ is also influenced by a pronounced A-site coordination sphere effect not observed in $\mathrm{BaNbO}_{2} \mathrm{~N}$, whereby local La- $(\mathrm{O}, \mathrm{N})_{12}$ coordination environments give rise to alternating $\mathrm{LaO}$ and $\mathrm{LaN}$ layers in the bulk material. Anion order was predicted to effect the redistribution of electrons upon anion vacancy creation to the cation sublattice. Diffusion barriers for $\mathrm{O}^{2-}$ vacancies in trans-ordered $\mathrm{BaNbO}_{2} \mathrm{~N}$ were found to be lower than those for $\mathrm{N}^{3-}$ vacancies, suggesting that stabilising transordered phases of $\mathrm{BaNbO}_{2} \mathrm{~N}$ will yield more effective retention of nitrogen content in this material. The reverse is the case for $\mathrm{LaNbON}_{2}$, with $\mathrm{N}^{3-}$ vacancy defects exhibiting more facile diffusion than $\mathrm{O}^{2-}$ vacancy defects. We believe these insights will aid the emergent understanding of defect engineering in mixed-anion perovskite oxynitride semiconductors, and specifically help facilitate strategies for stabilising their nitrogen content.
\end{abstract}

\section{Introduction}

Oxynitride materials have attracted recent interest for use in applications such as pigments, dielectrics, and optoelectronic materials. ${ }^{1}$ The overlap between the band gaps of many oxynitride materials and the solar spectrum also makes them potential water splitting photocatalysts that can operate in the visible-light region. ${ }^{2}$ Several niobium oxynitride perovskites, for instance, are promising candidate semiconductors for solar energy conversion owing to their wide visible light absorption. ${ }^{3-6}$

However, niobium oxynitrides are known to be more prone to defect formation during their synthesis via nitridation, compared to other perovskite oxynitrides (e.g. tantalum oxynitrides). This is a feature, rather than a flaw. Structural defects, such as zero-dimensional defects (e.g. vacancy, interstitial and

\footnotetext{
${ }^{a}$ School of Environmental \& Life Sciences, The University of Newcastle, Callaghan 2308, NSW, Australia. E-mail: alister.page@newcastle.edu.au

${ }^{b}$ School of Materials Science \& Engineering, PCFM Lab, Sun Yat-sen University, Guangzhou, 510275, P. R. China

$\dagger$ Electronic supplementary information (ESI) available: Relative energies of $\mathrm{LaNbO}_{2} \mathrm{~N}, \mathrm{BaNbON}_{2}$ anion orderings in the presence of vacancy defects, comparison of vacancy diffusion barrier heights as a function of supercell size. See DOI: $10.1039 / \mathrm{d} 1 \mathrm{ma} 00122 \mathrm{a}$
}

anti-site defects) and higher-dimensional defects (dislocations, grain boundaries, and volumetric defects) provide design parameters for modulating surface active sites, charge carrier mobilities, and band edge positions. ${ }^{7,8}$ of these, zero-dimensional point defects have the strongest link to material non-stoichiometry. ${ }^{7}$ Niobium oxynitrides are therefore ideal candidates for defect engineering. For instance, the enhanced conductivity of $\mathrm{BaNbO}_{2} \mathrm{~N}$ compared to $\mathrm{BaTaO}_{2} \mathrm{~N}$ is suspected to arise from defect concentration in the former. ${ }^{9}$ Niobium oxynitrides also have larger absorption tails extending in the visible region of the spectrum, which is indicative of nitrogen vacancies and reduced B-site cations, compared to their tantalum counterparts. ${ }^{9}$

However, engineering structural defects in perovskite oxynitride photocatalysts, such as $\mathrm{AM}(\mathrm{O}, \mathrm{N})_{3}(\mathrm{M}=\mathrm{Ti}, \mathrm{Zr}, \mathrm{Hf}, \mathrm{Ta}, \mathrm{Nb})$, in a controlled manner is complicated by the lack of long-range structural order in their crystal lattices. Such disorder is otherwise known as 'anion ordering'; despite exhibiting local ordering of anions within individual $\mathrm{MO}_{6-x} \mathrm{~N}_{x}$ octahedra, long-range structural order is lost due to the similarity between the $\mathrm{M}^{n+}-\mathrm{O}^{2-}$ and $\mathrm{M}^{n+}-\mathrm{N}^{3-}$ orbital overlaps, and hence bond lengths. The slightly larger $\mathrm{M}^{n+}-\mathrm{N}^{3-}$ bond length drives a predominantly cis-ordering in individual octahedra, giving rise to $\mathrm{N}^{3-}-\mathrm{M}^{n+}-\mathrm{N}^{3-}$ chains (or $\mathrm{O}^{2-}-\mathrm{M}^{n+}-\mathrm{O}^{2-}$ in the case of $\mathrm{LaTaON}_{2}$ and $\mathrm{LaNbON}_{2}$ ). ${ }^{10}$ 

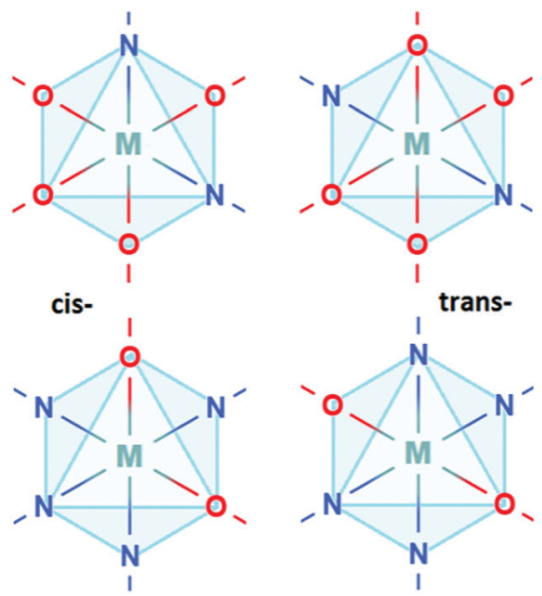

Fig. 1 Anion ordering in $\mathrm{M}-\mathrm{O}_{4} \mathrm{~N}_{2}$ and $\mathrm{M}-\mathrm{O}_{2} \mathrm{~N}_{4}$ octahedra.

Anion ordering induces local distortions ${ }^{11}$ in the crystal structure, influencing the ferroelectricity, ${ }^{12,13}$ bandgap $^{14,15}$ and effective charge carrier mobility of these materials. ${ }^{16-18}$

Anion ordering in oxynitride perovskites can be categorised into by the regimes of cis- or trans-ordering as illustrated in Fig. 1, as well as by the dimensionality the ordering travels in i.e. $2 \mathrm{D} / 3 \mathrm{D}$ for cis-ordering or $1 \mathrm{D} / 2 \mathrm{D}$ for trans-ordering. Recent research has focused on characterising the relative stabilities of these different regimes. While cis-ordering is the more stable for $\mathrm{d}^{0}$ oxynitrides, both regimes are sensitive to stoichiometry, strain, and interfacial effects. For instance, synthetic conditions and A-site cation substitution can yield fully 2D or 3D transorderings. ${ }^{16-20}$ For instance, increased $\mathrm{Sr}^{2+}$ doping in $\mathrm{CaTaO}_{2} \mathrm{~N}$ induces partial trans-ordering, with cis-ordering becoming energetically prohibitive due to lattice strain. ${ }^{21}$ Similarly, Vonruti et $a .^{22}$ have shown that $>4 \%$ compressive strain stabilizes trans-ordering in bulk $\mathrm{LaTiO}_{2} \mathrm{~N}$. Ninova et al. ${ }^{23}$ have predicted that, for $\mathrm{LaTiO}_{2} \mathrm{~N}$, surface layers can favour transordering due to the charge neutral stacking afforded by (LaN)$\left(\mathrm{TiO}_{2}\right)$ layers.

However, the impact of anion ordering on anion vacancy defect formation and anion vacancy diffusion remains largely unexplored in perovskite oxynitride photocatalysts.

Understanding this relationship is the first step towards controlling their defect engineering for enhanced photocatalysis. Here we address this issue by demonstrating how the stability and mobility of $\mathrm{O}^{2-}$ and $\mathrm{N}^{3-}$ vacancy point defects are influenced by local anion ordering in two representative niobium perovskite oxynitrides, $\mathrm{BaNbO}_{2} \mathrm{~N}$ and $\mathrm{LaNbON}_{2}$. Vacancy defect formation energies and migration barriers are investigated systematically as a function of both cis- and trans-ordering of $\mathrm{N}^{3-}-\mathrm{M}^{n+}-\mathrm{N}^{3-}$ $\left(\mathrm{BaNbO}_{2} \mathrm{~N}\right)$ and $\mathrm{O}^{2-}-\mathrm{M}^{n+}-\mathrm{O}^{2-}\left(\mathrm{LaNbON}_{2}\right)$ chains using accurate first-principles calculations. Cis-anion orderings are in general more energetically favourable than trans-orderings in these materials. However, there are secondary ordering effects that arise from the stoichiometric distribution of anions in the A-site cation coordination sphere, most prevalent for $\mathrm{LaNbON}_{2}$. We show below how both the stability and mobility of $\mathrm{O}^{2-}$ and $\mathrm{N}^{3-}$ vacancy defects are influenced by these two ordering effects.

\section{Computational methodology}

All calculations reported here employ the Perdew-BurkeErnzerhof generalized gradient approximation (GGA) exchangecorrelation functional, revised for solids (PBEsol), ${ }^{24}$ as implemented in the Vienna Ab initio Simulation Package (VASP) $\operatorname{code}^{25,26}$ using the projector augmented wave (PAW) method. ${ }^{27}$ A Hubbard- $U$ correction $^{28}$ has not been employed in this work, due to the non-trivial choice of selecting a $+U$ potential that is independent of the anion ordering and vacancy defect type. All calculations employ (core)/valence configurations of $\mathrm{Nb}$ : ([Ne]3s)/3p3d4s; $\mathrm{O}:([\mathrm{He}]) / 2 \mathrm{~s} 2 \mathrm{p} \mathrm{N}$ : ([He] $] / 2 \mathrm{~s} 2 \mathrm{p}$ and a plane wave energy cutoff of $450 \mathrm{eV}$. Crystal structures of $\mathrm{BaNbO}_{2} \mathrm{~N}$ and $\mathrm{LaNbON}_{2}$ at standard conditions were obtained from the International Crystal Structure Database (ICSD crystal IDs 434770 and 247261, respectively ${ }^{29}$ ), see Fig. 2. These structures are, respectively, of $P_{m \overline{3} m}$ and $P_{n m a}$ symmetry. However, these symmetries are only nominal; we show below that local anion orderings within each extended supercell induce differing degrees of three-dimensional anisotropy in these ideal lattice structures when they are fully relaxed. Monkhorst-Pack meshes consisting of $6 \times 6 \times 6 k$-points were used for the 40 atom $2 \times 2 \times 2 \mathrm{BaNbO}_{2} \mathrm{~N}$ supercell, while $4 \times 6 \times 4 k$-point meshes were used for 80 atom $2 \times 1 \times 2$ $\mathrm{LaNbON}_{2}$ supercell. Vacancy migration barriers were preoptimised using the nudged elastic band (NEB) algorithm ${ }^{30}$ to a force criterion of $0.1 \mathrm{eV} \AA^{-1}$, before being re-optimised the Climbing Image (CI-NEB) algorithm ${ }^{31}$ to a $0.05 \mathrm{eV}^{-1}$ criterion. Five images were used in both cases. ${ }^{31}$

The formation of a neutral oxygen vacancy in $\mathrm{BaNbO}_{2} \mathrm{~N}$ and $\mathrm{LaNbON}_{2}$ can be expressed in Kröger-Vink notation as,

$$
\mathrm{O}^{\times}+2 \mathrm{M}^{\times} \rightarrow 2 \mathrm{M}^{\prime}+\mathrm{V}_{\mathrm{O}}^{\bullet \bullet}+\frac{1}{2} \mathrm{O}_{2}
$$

and for a neutral nitrogen vacancy,

$$
\mathrm{N}^{\times}+3 \mathrm{M}^{\times} \rightarrow 3 \mathrm{M}^{\prime}+\mathrm{V}_{\mathrm{N}}^{\bullet \bullet \bullet}+\frac{1}{2} \mathrm{~N}_{2}
$$

The oxidation state of the $M$ cations in the vicinity of the defect will be determined by the localization of the electron density, which may vary between $2 \mathrm{M}^{\prime}+\mathrm{V}_{\mathrm{O}}^{\bullet \bullet}$ and $2 \mathrm{M}^{\prime}+\mathrm{V}_{\mathrm{O}}^{\bullet \times}$ for
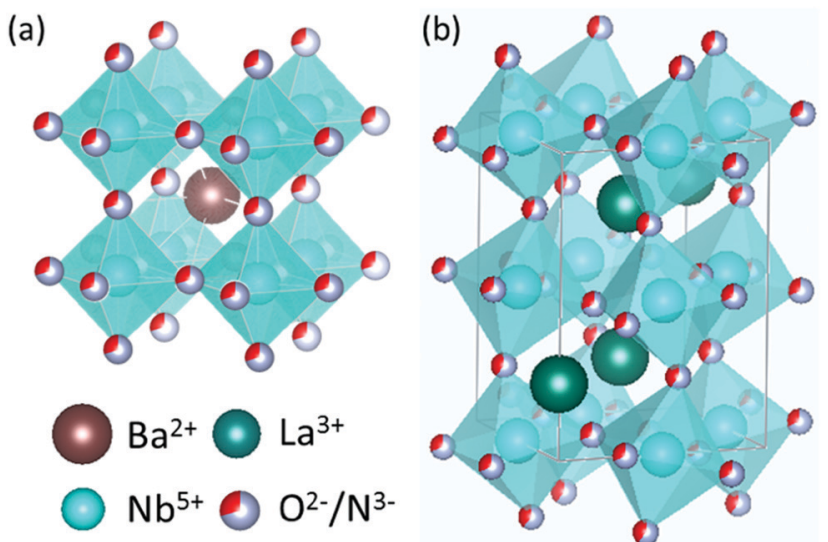

Fig. 2 Unit cells of (a) $P_{m \overline{3} m} \mathrm{BaNbO}_{2} \mathrm{~N}$ and (b) $P_{n m a} \mathrm{LaNbON}_{2}$. 
a neutral oxygen vacancy, and $3 \mathrm{M}^{\prime}+\mathrm{V}_{\mathrm{N}}^{\bullet \bullet \bullet}$ and $3 \mathrm{M}+\mathrm{V}_{\mathrm{N}}^{\bullet \times}$ for a neutral nitrogen vacancy. As $\mathrm{Nb}$ is capable of exhibiting a range of oxidation states $(+5$ to -1$)$, we reason that it will likely favour charged vacancies and reduced $\mathrm{Nb}$ cations in anion-deficient $\mathrm{BaNbO}_{2} \mathrm{~N}$ and $\mathrm{LaNbON}_{2}$. To this end we investigate the effect of anion ordering effect on electron localization and $\mathrm{Nb}$ oxidation via a DDEC6 charge decomposition analysis. ${ }^{32-34}$

For a neutral supercell, the anion vacancy formation energy, $E_{\mathrm{f}, \mathrm{vac}}$, can be obtained from the simplified expression: ${ }^{35}$

$$
E_{\mathrm{f}, \mathrm{vac}}=E_{\mathrm{def}}-E_{\mathrm{pris}}+\frac{1}{2} \mu_{i}
$$

where $E_{\text {def }}$ is the defective supercell, $E_{\text {pris }}$ is pristine supercell and $\mu_{i}$ chemical potential for the missing anion. In this work we seek only to establish how anion ordering influences anion vacancy formation (and vice versa), and do not consider the additional effects induced by oxygen/nitrogen-rich synthesis conditions. To this end we only employ a single nitrogen and oxygen chemical potential,obtained from the fitted elemental-phase reference energies (FERE) $\left(\mu_{\mathrm{O}}=-4.76 \mathrm{eV}\right.$ and $\left.\mu_{\mathrm{N}}=-8.51 \mathrm{eV}\right) .{ }^{36}$

\section{Results and discussion}

\section{Anion ordering in $\mathrm{Nb}$ perovskite oxynitrides}

$\mathrm{BaNbO}_{2}$ N. Fig. 3 illustrates each anion ordering considered here for $\mathrm{BaNbO}_{2} \mathrm{~N}$. This anion ordering is defined in terms of $\mathrm{N}^{3-}-\mathrm{Nb}^{5+}-\mathrm{N}^{3-}$ chains. The high symmetry of this structure
( $P_{m \overline{3} m}$ space group) enables an extensive search of anion orderings, including those that possess completely cis- or trans-ordered $\mathrm{N}^{3-}-\mathrm{Nb}^{5+}-\mathrm{N}^{3-}$ chains, as well as those that possess a mixture of both cis- and trans-orderings. Fig. 4a depicts the relative energies of these distinct anion orderings, compared to the lowest energy structure of $\mathrm{BaNbO}_{2} \mathrm{~N}$. The latter is the fully cis-C1 ordering, which has previously been found to be the most stable ordering in $\mathrm{LaTiO}_{2} \mathrm{~N},{ }^{14} \mathrm{CaTaO}_{2} \mathrm{~N}^{17}$ and $\mathrm{SrTaO}_{2} \mathrm{~N}^{15}$ Fig. 4a shows that several fully cis-orderings considered here lie within $0.3 \mathrm{eV}$ of C1, with the parallel ring structure C8 being the only exception. By comparison, local structures with fully trans-orderings (T1 and T2) are $>2 \mathrm{eV}$ less stable than the $\mathbf{C 1}$ ordering. Of these, the lowest energy fully trans configuration is T2, a $2 \mathrm{D}$ ordering comprised of stacked orthogonal layers of trans $-\mathrm{N}^{3-}-\mathrm{Nb}^{5+}-\mathrm{N}^{3-}$ chains. Interestingly, Fig. 4a shows that the partial trans-anion orderings considered here $(\mathbf{Q}$ and $\mathbf{H}$, which denote quarter and half trans-orderings, respectively) are all significantly lower in energy than the fully trans-configurations. Indeed, several quarter trans-orderings, such as $\mathbf{Q 1}$ and $\mathbf{Q 4}$, are predicted to be within $0.2 \mathrm{eV}$ of the lowest energy $\mathbf{C 1}$ ordering. This suggests that distortion caused by trans-chains can be stabilized to a point by adjacent cis-chains. These results suggest that stabilizing the formation of a fully trans-ordered phase in $\mathrm{BaNbO}_{2} \mathrm{~N}$ will be significantly more challenging than conversion from cis- to partially/fully trans-ordered phases, or forming mixed-phases comprising both cis- and trans-ordered regions. Of the ring \& loop configurations considered here (i.e. C7, C8, C9, Q2, H2),

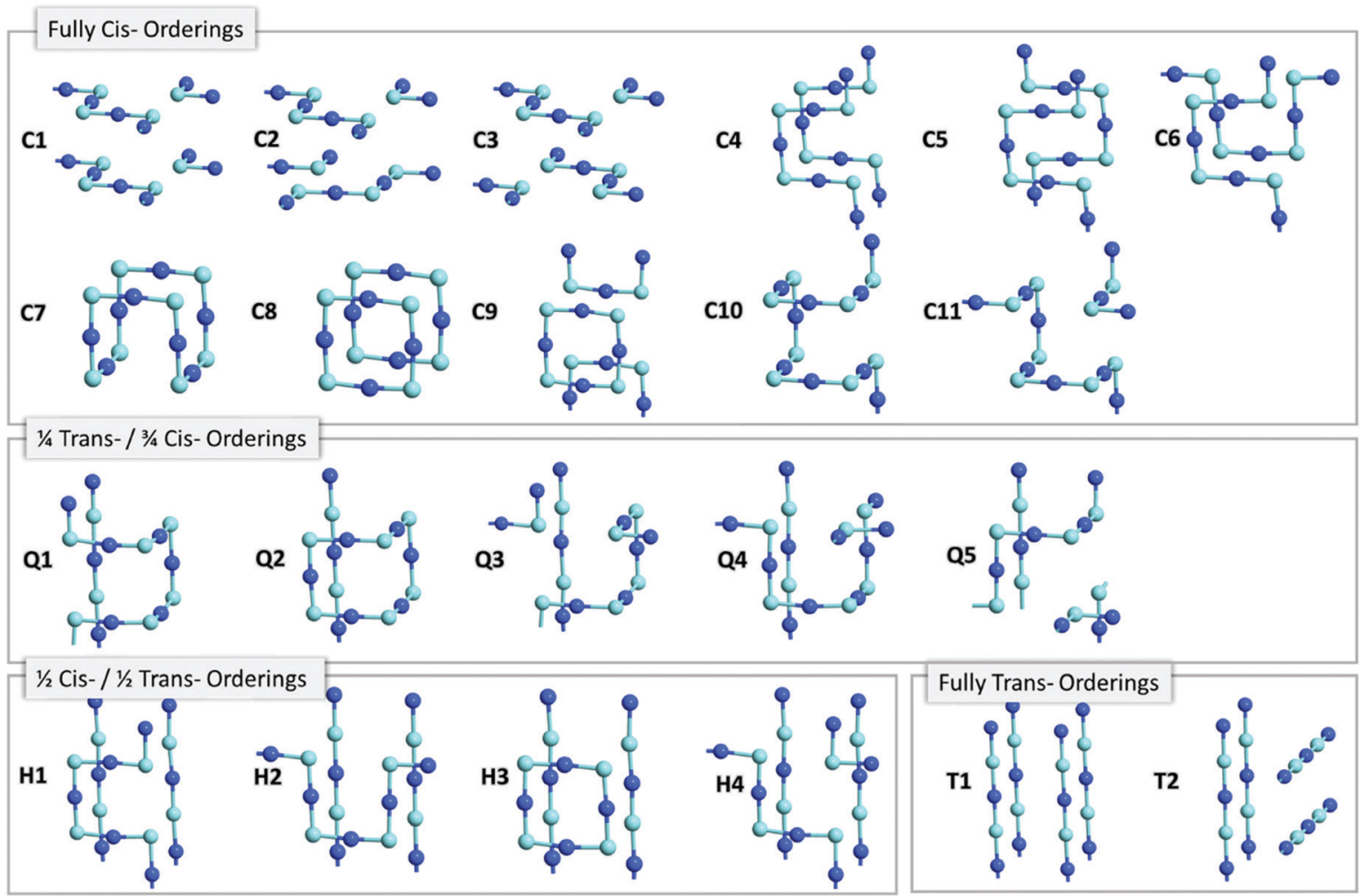

Fig. $3 \mathrm{~N}^{3-} \mathrm{Nb}^{5+}-\mathrm{N}^{3-}$ anion orderings in a $2 \times 2 \times 2 \mathrm{BaNbO}_{2} \mathrm{~N}$ supercell $\left(\mathrm{Ba}^{2+}\right.$ and $\mathrm{O}^{2-}$ atoms not shown for clarity). $\mathrm{N}^{3-}$ and $\mathrm{Nb}^{5+}$ are dark and light blue atoms, respectively. 
(a)

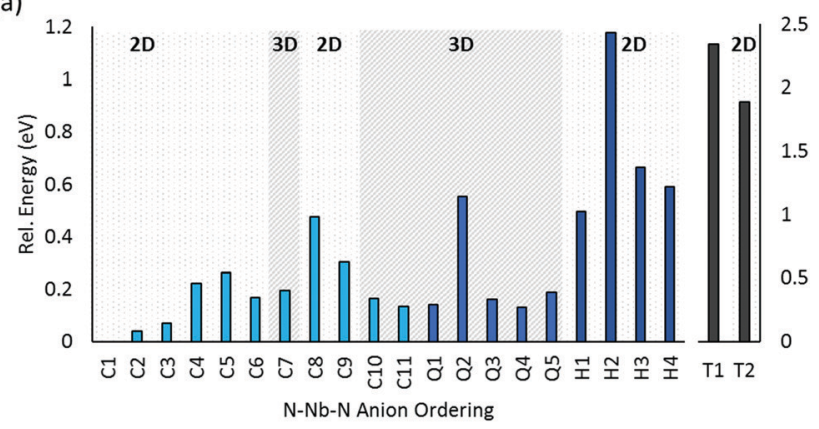

(b)

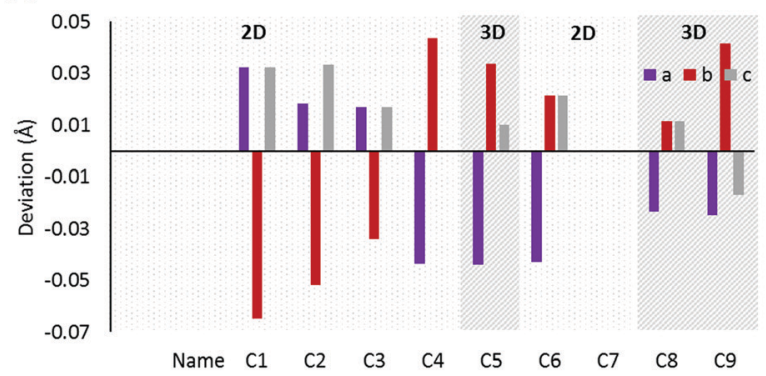

(c)

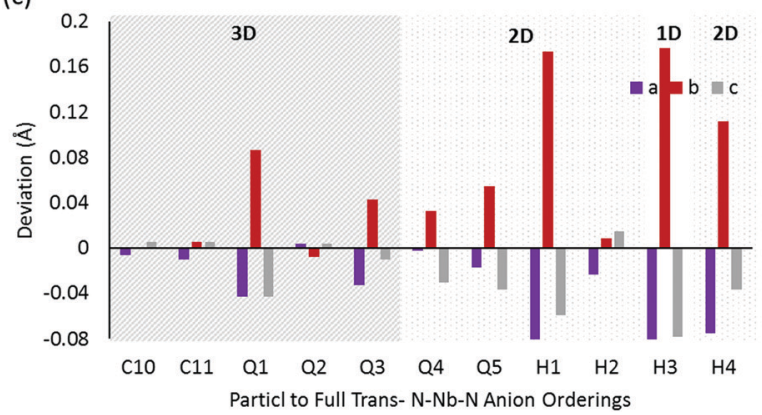

Fig. 4 (a) PBEsol energies of $\mathrm{BaNbO}_{2} \mathrm{~N}$ anion orderings relative to the lowest energy configuration for the $2 \times 2 \times 2$ supercell. Note the different scales for the cis- and trans-anion orderings. Deviation of lattice vectors from cubic symmetry for (b) cis-orderings and (c) Quarter, half and full trans-orderings in $\mathrm{BaNbO}_{2} \mathrm{~N}$.

Fig. 4a indicates that these orderings generally have higher energies than their counterparts. This suggests potentially unfavourable strain is produced when the $\mathrm{N}^{3-}-\mathrm{Nb}^{5+}-\mathrm{N}^{3-}$ chains 'loop back' upon themselves too tightly.

Fig. 4 also considers the relative stability of anion orderings in $\mathrm{BaNbO}_{2} \mathrm{~N}$ from the point of view of dimensionality, by highlighting which orderings are $2 \mathrm{D}$ and which are 3D. In general, cis-orderings are considered to be $2 \mathrm{D}$, in that they consist predominantly of $\mathrm{N}^{3-}-\mathrm{Nb}^{5+}-\mathrm{N}^{3-}$ chains aligned parallel with each other. Introducing locally trans-ordered $\mathrm{N}^{3-}-\mathrm{Nb}^{5+}$ $\mathrm{N}^{3-}$ chains, either partially ( $\mathbf{Q}$ configurations) or completely ( $T$ configurations) naturally increases the dimensionality of the ordering when 'twists' and 'loops' are present in the $\mathrm{N}^{3-}-\mathrm{Nb}^{5+}$ $\mathrm{N}^{3-}$ sublattice (see Fig. 3). Nevertheless, Fig. 4a shows that there is no clear relationship between dimensionality and stability here. For instance, the 2D fully cis-ordered structures are lowest in energy (e.g. C1, C2, C3) whereas the 2D halftrans-ordered structures $(\mathbf{H 1}, \mathbf{H 2}, \mathbf{H 3}, \mathbf{H 4})$ are less stable by up to $\sim 1.2 \mathrm{eV}$. Similarly, of the 3D orderings studied, the fully cis-C7, C10 and C11 and the quarter trans-Q1 and Q4 orderings have mostly very similar energies. Notably, the C10, Q1 and Q4 orderings are only $\sim 0.13 \mathrm{eV}$ higher in energy than the most stable $\mathbf{C 1}$ configuration. On the other hand, the dimensionality of the ordering has a clear relationship with the degree of lattice anisotropy, measured here via the deviation of each lattice constant from its value for a pristine $P_{m \overline{3} m}$ unit cell for each ordering (i.e. we define this value as the average of the $a, b$ and $c$ lattice constants in each case). Fig. $4 \mathrm{~b}$ and $c$ show that the 2D orderings considered here clearly induce greater anisotropy in the crystal lattice compared to $3 \mathrm{D}$ orderings, as anticipated. Indeed, lattice constant deviations correlate with the predominant alignment of $\mathrm{N}^{3-}-\mathrm{Nb}^{5+}-\mathrm{N}^{3-}$ chains in the $2 \times 2 \times 2$ supercell. For instance, for the most stable $\mathbf{C 1}$ ordering, the $\mathrm{N}^{3-}-\mathrm{Nb}^{5+}-\mathrm{N}^{3-}$ chains are aligned along the $a$ and $c$ lattice vectors (Fig. 3). This alignment causes equivalent expansion in $a$ and $c$ of $\sim 0.03 \AA$, while the lattice is compressed in $b$ approximately twofold, by $\sim-0.07 \AA$ (Fig. 4b). On the other hand, C7 yields an essentially isotropic $P_{m \overline{3} m}$ structure, and Fig. 3 shows that alignment of the $\mathrm{N}^{3-}-\mathrm{Nb}^{5+}-\mathrm{N}^{3-}$ chains are balanced exactly in all three directions. These results suggest that inducing lattice strain in $\mathrm{BaNbO}_{2} \mathrm{~N}$ will yield selectivity over anion ordering which is in line with recent studies for other oxynitride perovskites. ${ }^{16,21,22}$ We return to a discussion of how these orderings influence thermokinetic parameters of vacancy defects below.

Beyond dimensionality, Fig. $4 \mathrm{~b}$ and $\mathrm{c}$ show that these $\mathrm{BaNbO}_{2} \mathrm{~N}$ anion orderings fall into three distinct groups. The first of these comprise orderings that minimally distort the cubic symmetry of the ideal $\mathrm{BaNbO}_{2} \mathrm{~N}$ lattice, i.e. $a \approx b \approx c$. This isotropic group includes both high energy fully transorderings, as well as relatively stable $\mathbf{C 7}$ and Q2 orderings, and feature motifs in which $\mathrm{N}^{3-}-\mathrm{Nb}^{5+}-\mathrm{N}^{3-}$ chains form a closed loop within a $2 \times 2 \times 2$ extension of the $\mathrm{BaNbO}_{2} \mathrm{~N}$ lattice. The second group consists of orderings that yield anisotropic compression/expansion in a single dimension (i.e. two lattice constants essentially equal but distinct from the third), such as C1, C2, C3, H1, H3, and H4. The third group are those orderings that yield complete anisotropy in the sense that all three $\mathrm{BaNbO}_{2} \mathrm{~N}$ lattice constants are unique (e.g. C3, C4 Q3, Q4, Q5). In the second group, the C1, C2 \& C3 orderings (the three most stable orderings overall) are compressed in one axis and expanded in the other two. Interestingly, the half transorderings in this group (H1, H3, H4) all show the opposite trend, i.e. compression along two axes, and expansion in the third. These results suggest that cis-orderings in perovskite oxynitrides may be stabilised generally via in-plane lattice expansion, while trans-ordering may be stabilised via in-plane lattice compression. Indeed, Oka et $a .^{21}$ demonstrated the formation of a metastable trans-type anion orderings in $\mathrm{CaTaO}_{2} \mathrm{~N}$ via $\mathrm{Sr}^{2+} \mathrm{A}$-site doping, in which $\mathrm{N}^{3-}$ preferentially resided in axial sites in $\mathrm{TaO}_{4} \mathrm{~N}_{2}$ octahedra due to lattice strain induced by the A-site dopant. Similarly, Vonruti et $a .^{22}$ predicted the stabilization of parallel trans-ordering of $\mathrm{N}^{3-}$ anions (akin to T2) in $\mathrm{LaTiO}_{2} \mathrm{~N}$ when in-plane compressive strains greater than $4 \%$ were applied to the lattice. While an exhaustive strain engineering study is beyond the 
scope of this work, results presented here indicate that intermediate partial trans-orderings potentially impede the stabilization of fully trans-orderings via compressive in-plane strain, and so are likely an important consideration in strain engineering of perovskite oxynitrides more generally.

$\mathbf{L a N b O N}_{2}$. Fig. 5a illustrates the anion orderings considered here for $\mathrm{LaNbON}_{2}$. Here $\mathrm{NbO}_{2} \mathrm{~N}_{4}$ octahedra mean that anion ordering is best examined via $\mathrm{O}^{2-}-\mathrm{Nb}^{5+}-\mathrm{O}^{2-}$ chains, as opposed to $\mathrm{N}^{3-}-\mathrm{Nb}^{5+}-\mathrm{N}^{3-}$ chains. The lower orthorhombic symmetry here prevents an exhaustive study of anion orderings such as that presented above for the cubic $\mathrm{BaNbO}_{2} \mathrm{~N}$. Here we consider a set of chain structures that can provide an adequate representation of fully cis- or fully trans-anion orders in $\mathrm{LaNbON}_{2}$, and are commensurate with representation anion order sets considered in other DFT studies. ${ }^{15,17,22}$ Due to the lower symmetry of LaN$\mathrm{bON}_{2}$, we have also considered the chains in perpendicular orientations. The total orderings considered was ultimately limited to those configurations that could be constructed in the unit cell and subsequently extended into the $2 \times 1 \times 2$ supercell. Partial trans-orderings (akin to the $\mathbf{Q}$ and $\mathbf{H}$ structures considered for $\mathrm{BaNbO}_{2} \mathrm{~N}$ ) are not possible in the orthorhombic $\mathrm{LaNbON}_{2}$ unit cell without violating the $\mathrm{NbO}_{2} \mathrm{~N}_{4}$ octahedra selection rule, and so have not been considered.

Fig. 5b displays the relative energies of the different anion orderings for the $2 \times 1 \times 2 \mathrm{LaNbON}_{2}$ cell. It is clear that cisorderings are preferred over trans-orderings, consistent with results for $\mathrm{BaNbO}_{2} \mathrm{~N}$ presented in Fig. 4a. For $\mathrm{LaNbON}_{2}$ all trans-orderings considered here (LT1, LT2 \& LT3) are $>2 \mathrm{eV}$ less stable than the most stable cis-ordering, LC9. The latter ordering features 'zigzag' $\mathrm{O}^{2-}-\mathrm{Nb}^{5+}-\mathrm{O}^{2-}$ chains running through the $2 \times 1 \times 2$ supercell alternating between the (101) and (101) planes and perpendicular to the (011) and (01) directions. This zigzag motif is shared with the other stable anion orderings, LC1, LC2, and LC10, the only difference being the alignment of the motif within the crystal structure. For instance, in the LC10 ordering, the same zigzag $\mathrm{O}^{2-}-\mathrm{Nb}^{5+}-\mathrm{O}^{2-}$ chains are present in the $2 \times 1 \times 2$ supercell as those observed in the LC9 ordering. However, they are perpendicular to the (110) and (110) lattice directions, and as a result are $\sim 0.2 \mathrm{eV}$ higher in energy. Similarly, the LC1 and LC2 orderings also feature the zigzag $\mathrm{O}^{2-}-\mathrm{Nb}^{5+}-\mathrm{O}^{2-}$ chain motif, but perpendicular to the (101) and (101) lattice directions, respectively. Both orderings are only $\sim 0.15 \mathrm{eV}$ less stable than the most favourable ordering, LC9. The other cis-orderings considered here consist of different structural motifs and are less stable than the zigzag motif. These include the LC3, LC4, LC5 and LC6 orderings, all of which consist of $\mathrm{O}^{2-} \mathrm{Nb}^{5+}-\mathrm{O}^{2-}$ chains that create a repeating square-tooth pattern in the $2 \times 1 \times 2$ LaNbON $_{2}$ supercell and are $\sim 0.3-0.6 \mathrm{eV}$ less stable than LC9, and the ring-like LC7 and LC8 motifs, which are both $\sim 0.5 \mathrm{eV}$ less stable than LC9.

Fig. $5 \mathrm{~b}$ shows that the dimensionality of the ordering is also apparently unrelated to its stability. For instance, LC9 and LC10 are both $3 \mathrm{D}$ orderings, whereas all their cis-orderings of $\mathrm{O}^{2-}$ $\mathrm{Nb}^{5+}-\mathrm{O}^{2-}$ chains in $\mathrm{LaNbON}_{2}$ are $2 \mathrm{D}$. This is different from what was found in Fig. 4 for $\mathrm{BaNbO}_{2} \mathrm{~N}$, and suggests that controlling for $2 \mathrm{D}$ or $3 \mathrm{D}$ more ordering may not be possible (a)
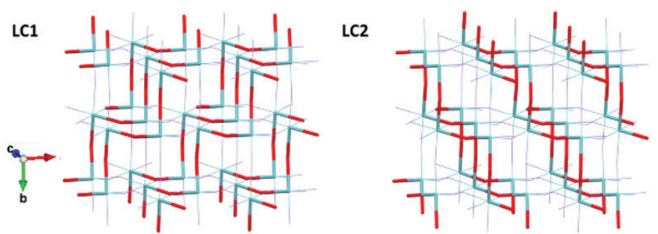

LC3

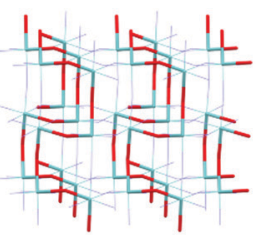

LC4

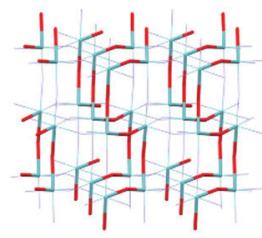

LC5

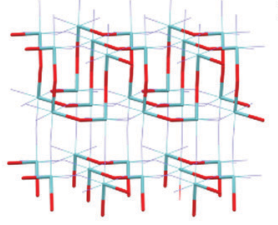

LC6

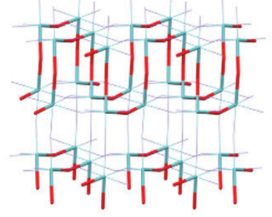

LC7

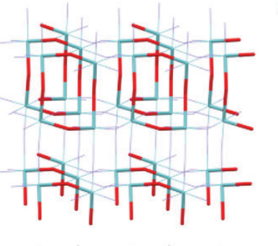

LC8

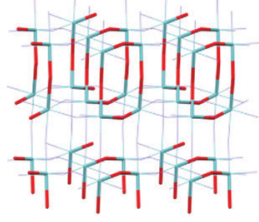

LC9

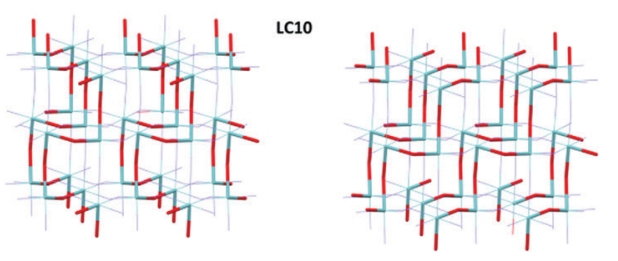

LT1

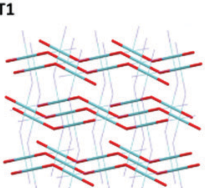

$1 \mathrm{~T} 2$

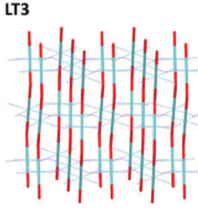

(b)

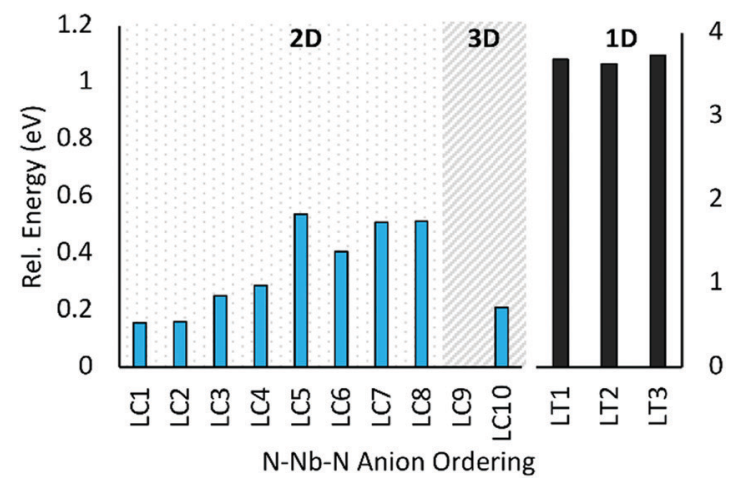

Fig. 5 (a) Representative $\mathrm{O}^{2-} \mathrm{Nb}^{5+}-\mathrm{O}^{2-}$ anion orderings in a $2 \times 1 \times 2$ LaNbON 2 supercell $\left(\mathrm{La}^{3+}\right.$ and $\mathrm{N}^{3-}$ not shown for clarity). $\mathrm{O}^{2-}$ and $\mathrm{Nb}^{5+}$ are red and light blue atoms, respectively. (b) PBEsol energies of $\mathrm{LaNbON}_{2}$ anion orderings shown in (a), relative to the lowest energy ordering LC9. 
in $\mathrm{LaNbON}_{2}$. Though we note that $2 \mathrm{D}-3 \mathrm{D}$ ordering transition temperatures have been observed in the comparable structure $\mathrm{LaTaON}_{2}{ }^{20}$

Further, comparison of Fig. 5b with equivalent results for $\mathrm{BaNbO}_{2} \mathrm{~N}$ (Fig. 3 and 4a), shows striking similarities between the relative stabilities of comparable $\mathrm{O}^{2-}-\mathrm{Nb}^{5+}-\mathrm{O}^{2-}$ orderings in $\mathrm{LaNbON}_{2}$ and $\mathrm{N}^{3-}-\mathrm{Nb}^{5+}-\mathrm{N}^{3-}$ orderings in $\mathrm{BaNbO}_{2} \mathrm{~N}$. For instance, cis-orderings featuring zigzag chain motifs are the most stable in both materials, and fully trans-anion orderings are significantly higher in energy (by $\sim 1 \mathrm{eV}$ ). Further, the ringlike and loop-like motifs are also energetically similar in both cases. For instance, LC3, LC4, LC5 and LC6 in $\mathrm{LaNbON}_{2}$ and C4, $\mathbf{C 5}$ and $\mathbf{C 6}$ in $\mathrm{BaNbO}_{2} \mathrm{~N}$ both feature repeating square tooth $\mathrm{O}^{2-}-\mathrm{Nb}^{5+}-\mathrm{O}^{2-}$ and $\mathrm{N}^{3-}-\mathrm{Nb}^{5+}-\mathrm{N}^{3-}$ motifs and have comparable relative energies $(\sim 0.3-0.4 \mathrm{eV})$. Similarly, the loop motifs in LC7 and LC8 for $\mathrm{LaNbON}_{2}$ and C8 and C9 in $\mathrm{BaNbO}_{2} \mathrm{~N}$ are structurally similar and $\sim 0.5-0.6 \mathrm{eV}$ higher in energy than the most favourable ordering.

Nevertheless, the $\mathrm{La}^{3+}$ and $\mathrm{Ba}^{2+}$ A-site cations also drive secondary ordering effects that differ between $\mathrm{BaNbO}_{2} \mathrm{~N}$ and $\mathrm{LaNbON}_{2}$. Comparing the $\mathrm{La}-(\mathrm{O}, \mathrm{N})_{12}$ coordination spheres between different orderings for $\mathrm{LaNbON}_{2}$, the lowest energy orderings (LC1-LC4 and LC9-10) all possess stoichiometric La- $\mathrm{O}_{4} \mathrm{~N}_{8}$ coordination spheres, whereas the LC5-LC8 orderings feature a mix of La- $\mathrm{O}_{6} \mathrm{~N}_{6}$ and $\mathrm{La}-\mathrm{O}_{2} \mathrm{~N}_{10}$ coordination spheres. This mix creates alternating $\mathrm{LaO}$ and LaN layers between $\mathrm{NbON}^{3-}$ sheets aligned perpendicular to the $b$-axis in the $2 \times$ $1 \times 2 \mathrm{LaNbON}_{2}$ supercell. There is a clear energetic difference between these two groups, with LC5-LC8 being $\sim 0.3 \mathrm{eV}$ higher on average than LC1-LC4 and LC9-10. This A-site effect is notably absent in the $\mathrm{BaNbO}_{2} \mathrm{~N}$ orderings, shown in Fig. 4 . While the most stable zigzag orderings in $\mathrm{BaNbO}_{2} \mathrm{~N}$ do indeed create an even distribution of $\mathrm{Ba}-\mathrm{O}_{8} \mathrm{~N}_{4}$ coordination spheres, other orderings like $\mathbf{C 6}$ \& $\mathbf{C 8}$ have energies 0.15-0.30 eV higher than orderings with comparable $\mathrm{Ba}-(\mathrm{O}, \mathrm{N})_{12}$ coordination sphere distributions. Thus, the $\mathrm{N}^{3-}-\mathrm{Nb}^{5+}-\mathrm{N}^{3-}$ chain ordering in the more densely packed $\mathrm{BaNbO}_{2} \mathrm{~N}$ supercell effectively masks the effect of the $\mathrm{Ba}-(\mathrm{O}, \mathrm{N})_{12}$ coordination sphere on the stability of the material.

\section{Vacancy defect formation energies in $\mathrm{Nb}$ perovskite oxynitrides}

We now consider the impact of anion order in $\mathrm{BaNbO}_{2} \mathrm{~N}$ and LaNbON $_{2}$ on the stability of vacancy defects in both $\mathrm{O}^{2-}$ and $\mathrm{N}^{3-}$ lattice positions. This is achieved via predicting the vacancy defect formation energies, $E_{\mathrm{f}, \mathrm{Vo}}$ and $E_{\mathrm{f}, \mathrm{VN}}$ (eqn (3)) at the 12 distinct anion positions in the respective supercells of these materials, for each specific anion ordering considered in the preceding discussion. These distinct positions 1-12 for the $2 \times 2 \times 2 \mathrm{BaNbO}_{2} \mathrm{~N}$ and $2 \times 1 \times 2$ LaNbON $_{2}$ supercells are depicted in Fig. 6a and $\mathrm{b}$.

$\mathbf{B a N b O}_{2} \mathbf{N} . E_{\mathrm{f}, \mathrm{Vo}}$ and $E_{\mathrm{f}, \mathrm{VN}}$ in $\mathrm{BaNbO}_{2} \mathrm{~N}$ for the 22 individual anion orderings introduced previously are shown in Fig. $6 \mathrm{c}$, and grouped according to their cis-/trans-character. This figure shows that nitrogen vacancy defects in $\mathrm{BaNbO}_{2} \mathrm{~N}$ have a smaller variation in formation energies in the presence of fully cis(2.24-2.50 eV) or fully trans-ordering (1.14-1.46 eV). By contrast, (a)

(c)
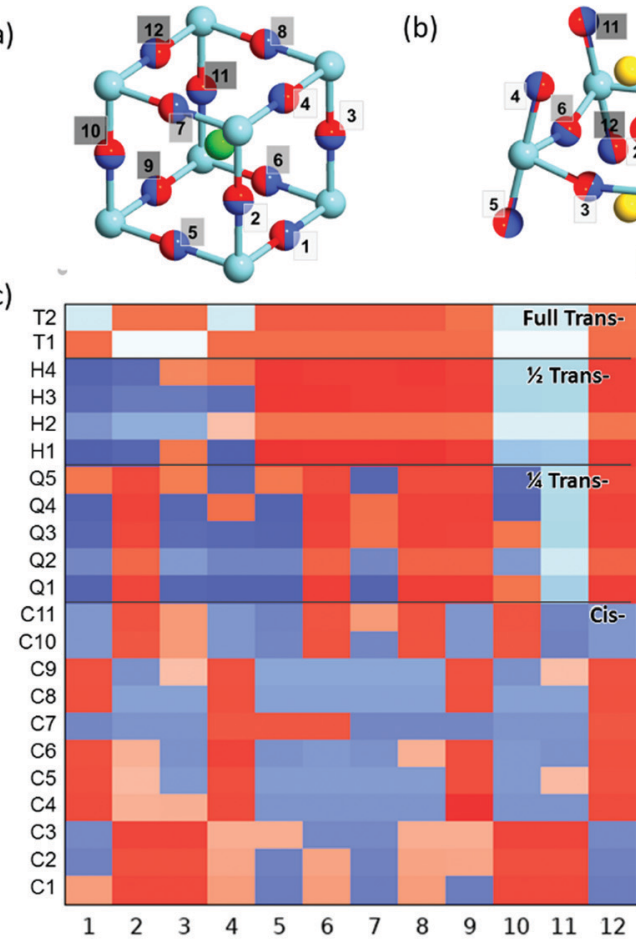

(d)

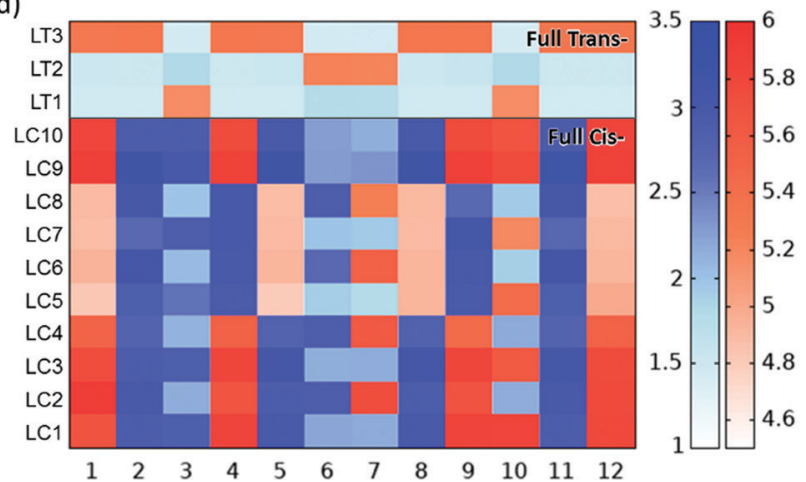

(b)
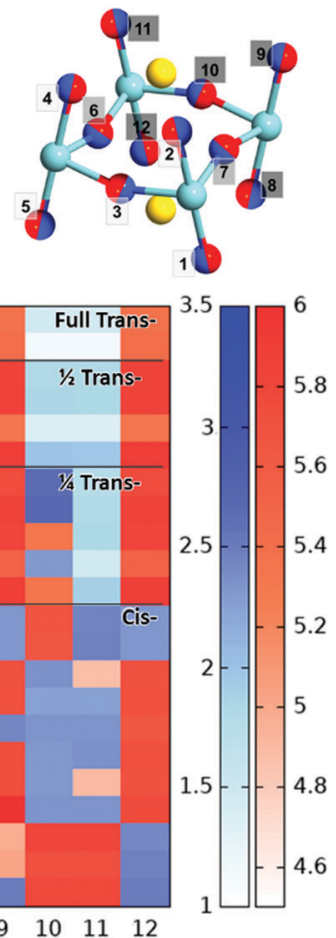

Fig. 6 ( $a$ and $b$ ) Depiction of the 12 unique anion positions in the centroid of the (a) $2 \times 2 \times 2 \mathrm{BaNbO}_{2} \mathrm{~N}$ and (b) $2 \times 1 \times 2 \mathrm{LaNbON}_{2}$ supercells. Light and dark blue spheres as in Fig. 3, red, green and yellow spheres represent oxygen, barium and lanthanum atoms respectively. (c and d) PBEsol vacancy formation energies $V_{N}$ and $V_{O}$ in positions 1-12 as a function of anion ordering for the (c) $2 \times 2 \times 2 \mathrm{BaNbO}_{2} \mathrm{~N}$ and (d) $2 \times 1 \times 2 \mathrm{LaNbON}_{2}$ supercells; red indicates an $\mathrm{O}^{2-}$ vacancy defect and blue indicates an $\mathrm{N}^{3-}$ vacancy defect at that position. The colour depth indicates the magnitude of the formation energies $V_{N}$ and $V_{O}$ (blue and red, respectively) in $\mathrm{eV}$, as indicated by the scale bar.

the range of $E_{\mathrm{f}, \mathrm{VN}}$ formation energies found for the quarter and half trans anion orderings (i.e. Q1-Q5 and H1-H4) is larger, $1.35-2.77 \mathrm{eV}$, presumably due to the greater structural anisotropy in these orderings, which gives rise to a greater number of distinct vacancy sites. Fig. $6 \mathrm{c}$ also shows that $\mathrm{V}_{\mathrm{N}}$ sites in local cis- or local trans-anion sites of the partial trans-orderings are $\sim 0.2 \mathrm{eV}$ higher in energy than if the whole ordering was cis or trans.

The $E_{\mathrm{f}, \mathrm{Vo}}$ formation energies vary quite significantly across the fully cis- (4.87-6.08 eV) and partial trans- $(4.87-5.98 \mathrm{eV})$ orderings, compared to fully trans- $(5.37-5.60 \mathrm{eV})$. This indicating that 
neutral oxygen vacancy formation energies are more sensitive to $\mathrm{N}^{3-}-\mathrm{Nb}^{5+}-\mathrm{N}^{3-}$ chain disorder, whereas nitrogen vacancy formation energies depend simply on whether the site is in a cis- or trans-chain. $\mathrm{N}^{3-}$ anions in trans $-\mathrm{N}^{3-}-\mathrm{Nb}^{5+}-\mathrm{N}^{3-}$ chains, whether they be partially or fully trans-, have a reduced $E_{\mathrm{f}, \mathrm{VN}}$ formation energy.

The question as to whether anion vacancies have the potential to affect local anion order in $\mathrm{BaNbO}_{2} \mathrm{~N}$ is considered in Fig. S1 $(\mathrm{ESI} \dagger)$, which shows the relative energies of these anion orderings in the presence of $\mathrm{V}_{\mathrm{O}}$ and $\mathrm{V}_{\mathrm{N}}$ defects. Compared to the defect-free relative energies in Fig. 4a, it can be seen in Fig. S1a (ESI $\dagger$ ) that in several cases anion vacancies likely influence the anion ordering. For instance, the quarter-trans orderings (Q1-5) become energetically indistinguishable from the cis (C1-11) orderings in the presence of a neutral oxygen vacancy defect. Additionally, the relative orderings in the presence of a neutral nitrogen vacancy defect (Fig. S1b, ESI $\dagger$ ) are significantly different from the defect-free orderings. While the cis C1-11 orderings are effectively equivalent in energy with and without a $\mathrm{V}_{\mathrm{N}}$ defect, the Q1-5 orderings are lowered by $\sim 0.5 \mathrm{eV}$, as the defect disrupts an $\mathrm{N}^{3-}-\mathrm{Nb}^{5+}-\mathrm{N}^{3-}$ chain, thus making them the most favourable ordering.

LaNbON $_{2} \cdot E_{\mathrm{f}, \mathrm{Vo}}$ and $E_{\mathrm{f}, \mathrm{VN}}$ in $\mathrm{LaNbON}_{2}$ for the 12 individual anion orderings introduced previously are shown in Fig. 6d, and grouped according to their cis-/trans-character. It is clear from this figure that $\mathrm{N}^{3-}$ anion vacancies form more easily in fully trans-ordered structures (LT1, LT2, LT3) than the cis-ordered LC structures. The LC5, LC6, LC7, and LC8 orderings are exceptions here, exhibiting $E_{\mathrm{f}, \mathrm{Vo}}$ values between only $4.82-5.54 \mathrm{eV}$ (the lowest values for all orderings considered here). We note that these are the orderings that yield alternating $\mathrm{LaO} / \mathrm{LaN}$ layers in $\mathrm{LaNbON}_{2} . E_{\mathrm{f}, \mathrm{VN}}$ values are notably higher than $E_{\mathrm{f}, \mathrm{Vo}}$ for the $\mathbf{L C}$ orderings. This means that $\mathrm{LaNbON}_{2}$ domains rich in these more stable cis-anion orderings are more likely to exhibit $\mathrm{O}^{2-}$ anion vacancies than $\mathrm{N}^{3-}$ vacancies. The same trend was noted above in our discussion of $\mathrm{BaNbO}_{2} \mathrm{~N}$ vacancy defects, indicating that the relative stabilities of $\mathrm{O}^{2-}$ and $\mathrm{N}^{3-}$ point vacancy defects in $\mathrm{AMO}_{2} \mathrm{~N} \& \mathrm{AMON}_{2}$ perovskite oxynitrides are potentially independent of the A-site cation and crystal symmetry. $E_{\mathrm{f}, \mathrm{VN}}$ formation energies predicted for $\mathrm{LaNbON}_{2}(1.74 \mathrm{eV}$ range) here are more sensitive to the local anion ordering, and thus exhibit a wider range than observed for $\mathrm{BaNbO}_{2} \mathrm{~N}$
(1.36 eV range). This is attributed to $\mathrm{N}^{3-}$ being the majority anion $\mathrm{LaNbON}_{2}$.

Finally, we consider whether or not $V_{O}$ and $V_{N}$ defects in $\mathrm{LaNbON}_{2}$ have the potential to influence the relative stabilities of these anion orderings. Fig. S2 (ESI $\dagger$ ) shows the relative energies of the anion orderings in the defective $\mathrm{LaNbON}_{2}$ structures. Compared to the defect-free relative energies in Fig. 5a and Fig. S2a (ESI $\dagger$ ) the presence of $\mathrm{V}_{\mathrm{O}}$ does not appreciably influence the relative stability of many of the orderings. However, the LC5-9 orderings become the most stable orderings, as they can be lowered by up to $\sim 0.2 \mathrm{eV}$. Fig. S2b (ESI $\dagger$ ) shows that, by comparison, $\mathrm{V}_{\mathrm{N}}$ defects are much more influential on the stabilities of these anion orderings, with all LC orderings being lowered by up to $\sim 0.5 \mathrm{eV}$. Nevertheless, the relative stabilities for all LC orderings become virtually indistinguishable in the presence of a $\mathrm{V}_{\mathrm{N}}$ defect.

\section{Vacancy defects and charge redistribution}

$\mathrm{Nb}$ is capable of exhibiting multiple low oxidation states. While our calculations enforce charge neutrality on the entire supercell, the region surrounding a vacancy defect is itself not necessarily guaranteed to be charge neutral due to electron density delocalisation. In turn, the oxidation states of $\mathrm{Nb}$ cations are not necessarily uniform throughout the supercell, since, despite the formal vacancy charge (i.e. $\mathrm{V}_{\mathrm{O}}^{\bullet \bullet}$ and $\mathrm{V}_{\mathrm{N}}^{\bullet \bullet}$ ) electron density delocalisation around the vacancy may lead to charge redistribution on neighbouring atoms. We examine this possibility in $\mathrm{BaNbO}_{2} \mathrm{~N}$ and $\mathrm{LaNbON}_{2}$ via the DDEC6 charge decomposition analysis presented in Table 1, which summarizes the partial atomic charges and charge redistribution in these materials following the formation of $\mathrm{V}_{\mathrm{O}}$ and $\mathrm{V}_{\mathrm{N}}$ defects. We limit our discussion to only the most thermodynamically stable cis/trans anion orderings for each material, established in the preceding discussion (i.e. $\mathbf{C 1}$ and $\mathbf{T} 2$ in $\mathrm{BaNbO}_{2} \mathrm{~N}$, and $\mathbf{L C 9}$ and LT2 for $\mathrm{LaNbON}_{2}$ ). Due to the size of the supercells used in this work, we have not verified these PBEsol results with higherlevel exchange correlation functionals, such as HSE06. ${ }^{37}$ We point out, however, that charges based on PBEsol have been shown to be in close agreement with higher level functionals for closely related transition metal oxide perovskites. ${ }^{38,39}$

For $\mathrm{BaNbO}_{2} \mathrm{~N}$, Table 1 shows that charge redistribution, due to delocalisation of the electron density around the defect site, leads to the 'true' charge of $\mathrm{V}_{\mathrm{O}}^{\bullet \bullet}$ and $\mathrm{V}_{\mathrm{N}}^{\bullet \bullet}$ defects being only

Table 1 DDEC6 average partial charges and charge redistribution (e) in $\mathrm{BaNbO}_{2} \mathrm{~N}$ and $\mathrm{LaNbON}_{2}$ due to 'neutral' $\mathrm{V}_{\mathrm{O}}$ and $\mathrm{V}_{\mathrm{N}}$ vacancy defects using PBEsol. The redistributed charge of an atom is defined as the difference between its DDEC 6 charges in the pristine and defective supercell; negative values indicate reduction in the presence of the defect. All values are averaged over all $\mathrm{O}^{2-} / \mathrm{N}^{3-}$ positions in each respective supercell. 'Adjacent' $\mathrm{Nb}$ cations are those that are coordinated to the anion removed to form the defect, 'other' $\mathrm{Nb}$ cations are all that are not

\begin{tabular}{|c|c|c|c|c|c|c|c|c|c|}
\hline & & \multicolumn{4}{|c|}{$\underline{\mathrm{BaNbO}_{2} \mathrm{~N}}$} & \multicolumn{4}{|c|}{$\underline{\mathrm{LaNbON}}_{2}$} \\
\hline & & $\mathrm{V}_{\mathrm{O}}$ & $\mathrm{V}_{\mathrm{N}}$ & $\mathrm{V}_{\mathrm{O}}$ & $\mathrm{V}_{\mathrm{N}}$ & $\mathrm{V}_{\mathrm{O}}$ & $\mathrm{V}_{\mathrm{N}}$ & $\mathrm{V}_{\mathrm{O}}$ & $\mathrm{V}_{\mathrm{N}}$ \\
\hline \multirow[t]{2}{*}{ Partial Charge } & Adjacent $\mathrm{Nb}$ & 1.89 & 1.73 & 1.93 & 1.84 & 1.78 & 1.64 & 1.64 & 1.89 \\
\hline & Other $\mathrm{Nb}$ & 2.21 & 2.20 & 2.20 & 2.17 & 2.08 & 2.08 & 2.09 & 2.08 \\
\hline Charge Redistribution & $\mathrm{Ba} / \mathrm{La}$ & -0.25 & -0.30 & -0.28 & -0.32 & -0.27 & -0.35 & -0.46 & -0.34 \\
\hline
\end{tabular}


$65-75 \%$ of the expected formal values. For instance, for the $\mathbf{C 1}$ ordering the total charge redistribution in the $\mathrm{BaNbO}_{2} \mathrm{~N}$ lattice around a $V_{O}$ defect is -1.51 e (rather than exactly -2 ), while for a $V_{N}$ defect this figure is -1.95 e (rather than exactly $-3 \mathrm{e}$ ). Charge redistribution is slightly smaller for the T2 ordering (1.43 and 1.79 e, respectively), indicating that anion vacancies near trans $-\mathrm{N}^{3-}-\mathrm{Nb}^{5+}-\mathrm{N}^{3-}$ anion configurations in $\mathrm{BaNbO}_{2} \mathrm{~N}$ lead to less charge redistribution away from the vacancy site in the cation sublattice. Table 1 also shows that, following the formation of a neutral oxygen or nitrogen vacancy defect in $\mathrm{BaNbO}_{2} \mathrm{~N}$, the oxidation state of a $\mathrm{Nb}$ cation depends on its vicinity to the defect. For instance, the average partial charge of adjacent $\mathrm{Nb}$ cations is $\sim 0.5$ e lower than those not coordinated to the vacancy. This is true for both the $\mathbf{C 1}$ and $\mathbf{T 2}$ anion orderings, although the effect is again marginally smaller for the latter $(\sim 0.3 \mathrm{e})$, likely for the same reason noted above.

Very similar trends are observed in Table 1 regarding partial charges of adjacent and uncoordinated $\mathrm{Nb}$ cations in $\mathrm{LaNbON}_{2}$. For instance, for the lowest energy LC9 ordering, Nb cations adjacent to $\mathrm{a} \mathrm{V}_{\mathrm{O}}$ defect are more reduced by $\sim 0.3 \mathrm{e}$, compared to $\mathrm{Nb}$ cations further away. Similarly, for a $\mathrm{V}_{\mathrm{N}}$ defect, the difference is $\sim 0.4$ e (adjacent $\mathrm{Nb}$ cations being more reduced). $\mathrm{Nb}$ partial charges in the LT2 ordering are analogous by comparison. As for $\mathrm{BaNbO}_{2} \mathrm{~N}$, Table 1 shows that the DDEC6 charges obtained using PBEsol are notably lower than the expected formal values. For instance, in the LC9 ordering, the LaNbON $_{2}$ lattice is reduced by only $1.06 \mathrm{e}\left(\mathrm{V}_{\mathrm{O}}\right)$ and $1.46 \mathrm{e}\left(\mathrm{V}_{\mathrm{N}}\right)$, respectively. However, a marked increase in charge distribution is observed for an oxygen vacancy defect in the LT2 ordering. Here, the $\mathrm{LaNbON}_{2}$ lattice is reduced by $2.17 \mathrm{e}$, which is comparable to the expected formal charge (i.e. -2). This is attributed to the effect of the ordering itself. While the pristine
LT2 and LC9 structures exhibit essentially equivalent average $\mathrm{Nb}$ partial charges (2.14 compared to 2.10, not shown in Table 1), the introduction of the $\mathrm{V}_{\mathrm{O}}$ defect disrupts a trans $-\mathrm{O}^{2-}-\mathrm{Nb}^{5+}-\mathrm{O}^{2-}$ chain in the LT2 structure, but leaves the chain in the cis LC9 ordering unperturbed. This reduces the $\mathrm{Nb}$ sublattice by $\sim 1 \mathrm{e}$ $(-1.71$ e) more in LT2, compared to LC9 ordering (-1.06 e).

\section{Vacancy diffusion barriers in Nb perovskite oxynitrides}

With the thermochemical stability of $\mathrm{V}_{\mathrm{N}}^{\bullet \bullet}$ and $\mathrm{V}_{\mathrm{O}}^{\bullet \bullet}$ vacancy defects in $\mathrm{BaNbO}_{2} \mathrm{~N}$ and $\mathrm{LaNbON}_{2}$ now established, we conclude our discussion by considering the impact of anion ordering on vacancy diffusion kinetics in these materials. Fig. 7 presents diffusion pathways of $\mathrm{V}_{\mathrm{N}}^{\bullet \bullet \bullet}$ and $\mathrm{V}_{\mathrm{O}}^{\bullet \bullet}$ vacancy defects in $\mathrm{BaNbO}_{2} \mathrm{~N}$ and $\mathrm{LaNbON}_{2}$ elucidated using the CI-NEB method. Due to the expense of this approach, we consider defect diffusion in only the most stable cis- and trans-anion orderings (i.e. the most relevant), and only those diffusion pathways corresponding to vacancies moving between adjacent positions in the crystal lattice (i.e. the distinct positions defined in Fig. 6a and b). For $\mathrm{BaNbO}_{2} \mathrm{~N}$ we assess the accuracy of diffusion barriers obtained with the $2 \times$ $2 \times 2$ supercell by comparing them with barriers obtained with a $3 \times 3 \times 3$ supercell in Fig. S3 and Table S3 (ESI $\dagger$ ). This comparison indicates that shorter diffusion pathways (rotation about a $\mathrm{Nb}$ ion) are significantly less sensitive to supercell size than longer pathways (shifting to other $\mathrm{Nb}$ ion coordination spheres), as anticipated. Importantly, Table S3 (ESI $\dagger$ ) indicates that the trends in vacancy diffusion barriers found using the $2 \times$ $2 \times 2$ supercell are reproduced with the $3 \times 3 \times 3$ supercell. The longer diffusion pathways in $\mathrm{LaNbON}_{2}$ are orientated to the $a / c$ plane in the $2 \times 1 \times 2$ orthorhombic supercell, and so it is likely they are already converged with respect to the $a / c$ dimensions of the supercell. (a)
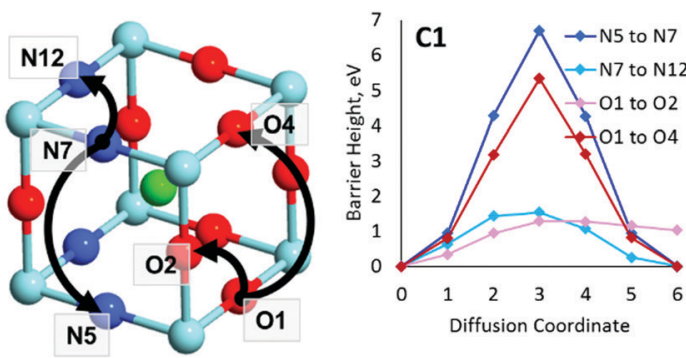

(c)
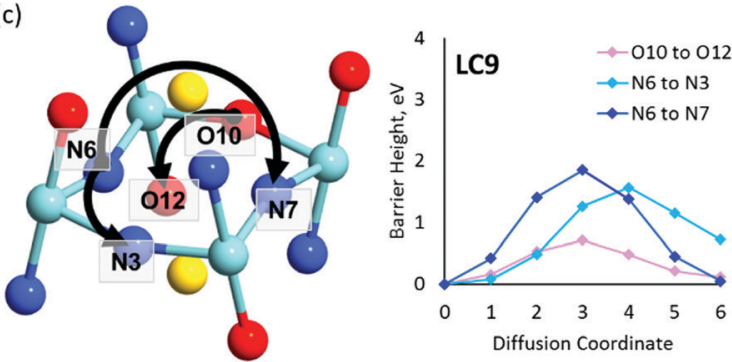
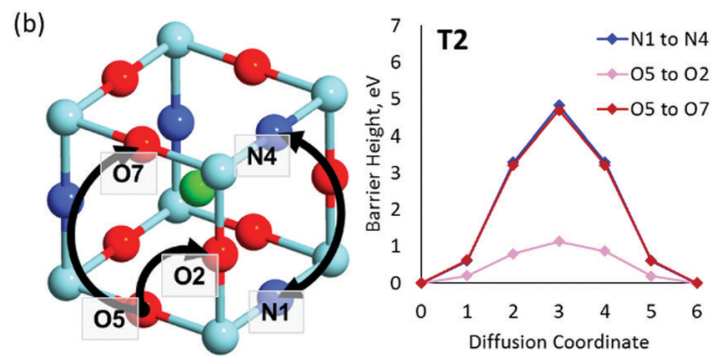

(d)

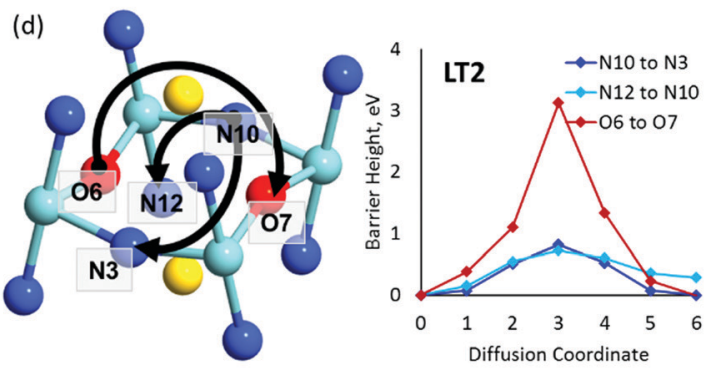

Fig. $7 \quad V_{N} \& V_{O}$ diffusion pathways and associated PBEsol diffusion barriers (eV) for the most stable cis-and trans-anion orderings in (a and b) BaNbO ${ }_{2} \mathrm{~N}$ and (c and d) $\mathrm{LaNbON}_{2}$. (a) $\mathrm{BaNbO}_{2} \mathrm{~N}$, C1 ordering, (b) $\mathrm{BaNbO}_{2} \mathrm{~N}$, T2 ordering, (c) LaNbON 2 , LC9 ordering, (d) LaNbON $\mathrm{L}_{2}$ LT1 ordering. All diffusion pathways were calculated in the complete $3 \times 3 \times 3$ and $2 \times 1 \times 2$ supercells, respectively, however only the core region is depicted here for clarity. Anion positions are defined in Fig. $5 \mathrm{a}$ and b for $\mathrm{BaNbO}_{2} \mathrm{~N}$ and $\mathrm{LaNbON}$, respectively. 
$\mathbf{B a N b O}_{2} \mathbf{N}$. Diffusion pathways for $\mathrm{V}_{\mathrm{N}}^{\bullet \bullet \bullet}$ and $\mathrm{V}_{\mathrm{O}}^{\bullet \bullet}$ vacancies in the most stable $\mathbf{C 1}$ and $\mathbf{T} 2$ orderings of $\mathrm{BaNbO}_{2} \mathrm{~N}$ and the corresponding energy barriers are presented in Fig. 7a and $\mathrm{b}$. There are two distinct pathways for each vacancy type; the defect can rotate positions about $\mathrm{a} \mathrm{Nb}^{5+}$ cation $(\mathrm{O} 1 \rightarrow \mathrm{O} 2$, $\mathrm{N} 7 \rightarrow \mathrm{N} 12$ in C1 (Fig. 7a); O5 $\rightarrow$ O2 in T2 (Fig. 7b)) or the defect can migrate to a non-adjacent position, shifting to a different $\mathrm{Ta}^{5+}$ cation but rotating about the $\mathrm{Ba}^{2+}$ cation $(\mathrm{O} 1 \rightarrow \mathrm{O} 4$, N7 $\rightarrow$ N5 in C1 (Fig. 7a); O5 $\rightarrow$ O7, N1 $\rightarrow$ N4 in T2 (Fig. 7b)). The latter of these pathways is obviously the longest and so is anticipated to correlate with the highest diffusion barrier. Fig. 7a and b show that this is the case; $\mathrm{V}_{\mathrm{O}}^{\bullet \bullet}$ and $\mathrm{V}_{\mathrm{N}}^{\bullet \bullet \bullet}$ migration between non-adjacent sites is impeded by barriers ranging from $5.33 \mathrm{eV}$ to $6.70 \mathrm{eV}$ while rotation of the defect to adjacent positions around the $\mathrm{Nb}^{5+}$ cation corresponds to a barrier of only $1.13 \mathrm{eV}$ to $1.53 \mathrm{eV}$. Fig. 7a and b also shows that, in general, $\mathrm{V}_{\mathrm{O}}^{\bullet \bullet}$ diffusion are barriers around $\sim 25 \%$ lower than the analogous diffusion pathways for $\mathrm{V}_{\mathrm{N}}^{\bullet \bullet \bullet}$. $\mathrm{V}_{\mathrm{N}}^{\bullet \bullet}$ diffusion in the less stable $\mathbf{T} 2$ ordering is further impeded by the fact that it can only occur between adjacent $\mathrm{NbO}_{4} \mathrm{~N}_{2}$ octahedra, and not via rotation around an individual $\mathrm{Nb}^{5+}$ cation, which would correspond to a lower barrier. Thus, despite being more stable in $\mathrm{BaNbO}_{2} \mathrm{~N}, \mathrm{O}^{2-}$ vacancy defects are expected to undergo more facile diffusion, most notably in trans-ordered phases, impeding control over long range ordering in this material. We note also that these findings are consistent with our recent study of TaON, in which it was shown that diffusion barriers for $\mathrm{V}_{\mathrm{O}}^{\bullet \bullet}$ migration are potentially low enough to displace nitrogen content from the oxynitride. ${ }^{40}$ Thus, it is suggested that stabilising trans-ordered phases of $\mathrm{BaNbO}_{2} \mathrm{~N}$ is likely to yield more effective retention of nitrogen content in this material, even if long-range order cannot be controlled.

$\mathbf{L a N b O N}_{2}$. Diffusion pathways for $\mathrm{V}_{\mathrm{N}}^{\bullet \bullet \bullet}$ and $\mathrm{V}_{\mathrm{O}}^{\bullet \bullet}$ vacancies in the lowest energy cis- and trans-ordered structures of $\mathrm{LaNbON}_{2}$, LC9, and LT1, and the corresponding energy barriers are presented in Fig. 7c and d. In the LC9 cis-ordering, Fig. 7c, $\mathrm{O}^{2-}$ vacancies can only diffuse via the $\mathrm{O} 10 \rightarrow \mathrm{O} 12$ pathway, while $\mathrm{N}^{3-}$ vacancies have two possibilities for migration via rotation around $\mathrm{a} \mathrm{Nb}^{5+}$ cation $(\mathrm{N} 6 \rightarrow \mathrm{N} 3)$ and between non-adjacent $(\mathrm{N} 6 \rightarrow \mathrm{N} 7)$ sites that cross the cavity between $\mathrm{La}^{3+}$ ions. In the latter case the defect is shifting to a separate $\mathrm{NbO}_{2} \mathrm{~N}_{4}$ octahedron while rotating is coordinating about the local $\mathrm{La}-(\mathrm{O}, \mathrm{N})_{12}$ coordinate sphere. In the less stable LT1 trans-ordering, Fig. 7d, again $\mathrm{O}^{2-}$ vacancies can only migrate via a single long pathway (O6 $\rightarrow$ O7) since rotation about an individual $\mathrm{Nb}^{5+}$ cation to another $\mathrm{O}^{2-}$ site isn't possible, whereas $\mathrm{N}^{3-}$ vacancies can migrate via the N12 $\rightarrow \mathrm{N} 10$ pathway and along the longer non-adjacent N10 $\rightarrow$ N3 pathway.

Consistent with $\mathrm{BaNbO}_{2} \mathrm{~N}$, the $\mathrm{V}_{\mathrm{O}}^{\bullet \bullet}$ diffusion barriers are seen to be sensitive to whether the diffusion path is between adjacent anion positions or non-adjacent positions with $\mathrm{O} 10 \rightarrow$ O12 in LC9 predicted to have a barrier height of $0.71 \mathrm{eV}$, while O6 $\rightarrow$ O7 in LT2 is $3.12 \mathrm{eV}$. However, the difference in the $\mathrm{V}_{\mathrm{N}}^{\bullet \bullet \bullet}$ diffusion barriers for adjacent and non-adjacent paths is greatly reduced compared to $\mathrm{BaNbO}_{2} \mathrm{~N}$. In LC9 the adjacent pathway height is $1.56 \mathrm{eV}$ with the non-adjacent path barrier only $0.29 \mathrm{eV}$ higher, while in LT2 the adjacent $\mathrm{V}_{\mathrm{N}}^{\bullet \bullet}$ diffusion is now 0.72 , with the non-adjacent barrier merely $0.82 \mathrm{eV}$.
Interestingly, the kinetic stability of $\mathrm{N}^{3-}$ vacancy defects in $\mathrm{LaNbON}_{2}$ is significantly reduced from that observed in $\mathrm{BaNbO}_{2} \mathrm{~N}$. In particular, in the less stable LT1 trans-ordering, $\mathrm{N}^{3-}$ vacancies diffuse more easily than $\mathrm{O}^{2-}$ vacancies. The origin of this reduction is a cooperative effect in which the migrating $\mathrm{V}_{\mathrm{N}}^{\bullet \bullet \bullet}$ vacancy partially displaces an adjacent $\mathrm{O}^{2-}$ anion. The additional space created by this displacement gives rise to an asymmetric path, in which the $\mathrm{V}_{\mathrm{N}}^{\bullet \bullet \bullet}$ vacancy does not pass directly between adjacent $\mathrm{La}^{3+}$ cations along the diffusion pathway. In doing so, the migrating $\mathrm{V}_{\mathrm{N}}^{\bullet \bullet \bullet}$ vacancy partially displaces an adjacent $\mathrm{O}^{2-}$ anion and so reduces the diffusion barrier.

\section{Conclusions}

The impact of anion ordering on defect engineering in perovskite oxynitride photocatalysts remains largely unexplored, despite it being the necessary first step towards controlling defects in these materials for improved photocatalytic performance. Using accurate first-principles calculations, we have presented an extensive investigation of cis- and trans-anion orderings in two exemplar perovskite oxynitrides, $\mathrm{BaNbO}_{2} \mathrm{~N}$ and $\mathrm{LaNbON}_{2}$, and examined how these orderings influence (1) overall stability, (2) the stability of $\mathrm{N}^{3-}$ and $\mathrm{O}^{2-}$ point defects and (3) the mobility of these defects through the bulk material. Analysis of partial charges with DDEC6 confirmed that the anion ordering influenced the degree of $\mathrm{e}^{-}$redistribution to adjacent and outer $\mathrm{Nb}$ cations from the creation of an anion vacancy.

For $\mathrm{BaNbO}_{2} \mathrm{~N}$, our results show clearly that fully cis-anion orderings are more stable than fully trans-anion orderings. The influence of these orderings on the cubic lattice vectors however demonstrates differing degrees of anisotropy. This ultimately has implications for strain engineering in this oxynitride; specifically, it may be difficult to stabilize the fully trans-orderings in $\mathrm{BaNbO}_{2} \mathrm{~N}$, over half-trans or fully-cis orderings, via in-plane compression of the crystal lattice. In $\mathrm{LaNbON}_{2}, 2 \mathrm{D} \& 3 \mathrm{D}$ orderings have comparable energies, meaning that mixed dimensionality in anion ordering may be more prevalent in this material. However, our results show the presence of a secondary 'A-site coordination sphere effect' influencing the stability of different orderings in $\mathrm{LaNbON}_{2}$, where the stoichiometric distribution of $\mathrm{O}^{2-}$ and $\mathrm{N}^{3-}$ in $\mathrm{La}-(\mathrm{O}, \mathrm{N})_{12}$ gives rise to obvious differences in the relative energies of different fully cis-anion orderings. This effect is less obvious in $\mathrm{BaNbO}_{2} \mathrm{~N}$, which we contend is due to the more densely packed lattice having a higher degree of symmetry, which masks any $\mathrm{Ba}-(\mathrm{O}, \mathrm{N})_{12}$ coordination sphere on the overall stability of the material. Vacancy defect formation energies for $\mathrm{BaNbO}_{2} \mathrm{~N}$ indicate that domains rich in cis-anion orderings (the most favourable) may preferentially stabilise $\mathrm{O}^{2-}$ anion vacancies less uniformly in their structure, whereas $\mathrm{N}^{3-}$ anion vacancies are less likely. On the other hand, $\mathrm{O}^{2-}$ defect formation energies in $\mathrm{LaNbON}_{2}$ are more sensitive to the local anion ordering, than its overall cis- or trans-character. This is attributed to the effect of the $\mathrm{La}^{3+} \mathrm{A}$-site cation, which for particular orderings considered here gives rise to alternating layers of $\mathrm{LaO}$ and $\mathrm{LaN}$ that reduce significantly $\mathrm{O}^{2-}$ vacancy defect formation energy. 
For $\mathrm{BaNbO}_{2} \mathrm{~N}, \mathrm{~V}_{\mathrm{O}}^{\bullet \bullet}$ diffusion barriers were found to be lower than $\mathrm{V}_{\mathrm{N}}^{\bullet \bullet \bullet}$, most notably in trans-ordered phases. It is predicted that this will impede control over long range ordering in $\mathrm{BaNbO}_{2} \mathrm{~N}$. Stabilising trans-ordered phases of this material is thus predicted to yield more effective retention of nitrogen content in its structure, irrespective of long-range anion order. In this sense, $\mathrm{BaNbO}_{2} \mathrm{~N}$ bears some resemblance to TaON, for which $\mathrm{V}_{\mathrm{O}}^{\bullet \bullet}$ migration barriers are potentially low enough to displace nitrogen content from the oxynitride. ${ }^{40}$ The reverse is observed true for $\mathrm{V}_{\mathrm{N}}^{\bullet \bullet \bullet}$ in $\mathrm{LaNbON}_{2}$, i.e. $\mathrm{N}^{3-}$ vacancy defects exhibit lower diffusion barriers than $\mathrm{O}^{2-}$ vacancy defects. $\mathrm{V}_{\mathrm{N}}$ diffusion via both adjacent and non-adjacent pathways in $\mathrm{LaNbON}_{2}$ benefits from the lower orthorhombic symmetry. In part this is due to the disruption the migrating $\mathrm{N}^{3-}$ defect has on the local coordination environment; when rotating around a local $\mathrm{La}^{3+}$ cation the $\mathrm{N}^{3-}$ anion vacancy partially displaces an adjacent $\mathrm{O}^{2-}$ ion to lower the diffusion barrier.

\section{Conflicts of interest}

There are no conflicts to declare.

\section{Acknowledgements}

This research was undertaken with the assistance of resources provided at the NCI National Facility systems at the Australian National University, through the National Computational Merit Allocation Scheme supported by the Australian Government. AJP acknowledges support from the Australian Research Council (INTERSECT, LE170100032). JJB acknowledges support from an Australian Government RTP Scholarship.

\section{Notes and references}

1 T. Takata and K. Domen, Dalton Trans., 2017, 46, 10529-10544.

2 M. Ahmed and G. Xinxin, Inorg. Chem. Front., 2016, 3, 578-590.

3 B. Siritanaratkul, K. Maeda, T. Hisatomi and K. Domen, ChemSusChem, 2011, 4, 74-78.

4 D. Y. Wan, Y. L. Zhao, Y. Cai, T. C. Asmara, Z. Huang, J. Q. Chen, J. Hong, S. M. Yin, C. T. Nelson, M. R. Motapothula, B. X. Yan, D. Xiang, X. Chi, H. Zheng, W. Chen, R. Xu, Ariando, A. Rusydi, A. M. Minor, M. B. H. Breese, M. Sherburne, M. Asta, Q. H. Xu and T. Venkatesan, Nat. Commun., 2017, 8, 15070.

5 X. Xu, C. Randorn, P. Efstathiou and J. T. S. Irvine, Nat. Mater., 2012, 11, 595-598.

6 K. Maeda, M. Higashi, B. Siritanaratkul, R. Abe and K. Domen, J. Am. Chem. Soc., 2011, 133, 12334-12337.

7 J. Liu, Z. Wei and W. Shangguan, ChemCatChem, 2019, 11, 6177-6189.

8 S. Bai, N. Zhang, C. Gao and Y. Xiong, Nano Energy, 2018, 53, 296-336.

9 Y.-I. Kim, P. M. Woodward, K. Z. Baba-Kishi and C. W. Tai, Chem. Mater., 2004, 16, 1267-1276.

10 M. Yang, J. Oró-Solé, J. A. Rodgers, A. B. Jorge, A. Fuertes and J. P. Attfield, Nat. Chem., 2010, 3, 47.

11 A. Fuertes, J. Mater. Chem., 2012, 22, 3293-3299.
12 D. Oka, Y. Hirose, H. Kamisaka, T. Fukumura, K. Sasa, S. Ishii, H. Matsuzaki, Y. Sato, Y. Ikuhara and T. Hasegawa, Sci. Rep., 2014, 4, 4987.

13 Y. Hinuma, H. Moriwake, Y.-R. Zhang, T. Motohashi, S. Kikkawa and I. Tanaka, Chem. Mater., 2012, 24, 4343-4349.

14 N. Vonrüti and U. Aschauer, Phys. Rev. Mater., 2018, 2, 105401.

15 A. Ziani, C. Le Paven, L. Le Gendre, F. Marlec, R. Benzerga, F. Tessier, F. Cheviré, M. N. Hedhili, A. T. Garcia-Esparza, S. Melissen, P. Sautet, T. Le Bahers and K. Takanabe, Chem. Mater., 2017, 29, 3989-3998.

16 H. Johnston, A. P. Black, P. Kayser, J. Oró-Solé, D. A. Keen, A. Fuertes and J. P. Attfield, Chem. Commun., 2018, 54, 5245-5247.

17 A. Kubo, G. Giorgi and K. Yamashita, Chem. Mater., 2017, 29, 539-545.

18 P. J. Camp, A. Fuertes and J. P. Attfield, J. Am. Chem. Soc., 2012, 134, 6762-6766.

19 S. G. Ebbinghaus, H.-P. Abicht, R. Dronskowski, T. Müller, A. Reller and A. Weidenkaff, Prog. Solid State Chem., 2009, 37, 173-205.

20 L. Clark, J. Oró-Solé, K. S. Knight, A. Fuertes and J. P. Attfield, Chem. Mater., 2013, 25, 5004-5011.

21 D. Oka, Y. Hirose, F. Matsui, H. Kamisaka, T. Oguchi, N. Maejima, H. Nishikawa, T. Muro, K. Hayashi and T. Hasegawa, ACS Nano, 2017, 11, 3860-3866.

22 N. Vonrüti and U. Aschauer, Phys. Rev. Lett., 2018, 120, 046001.

23 S. Ninova and U. Aschauer, J. Mater. Chem. A, 2017, 5, 11040-11046.

24 J. P. Perdew, A. Ruzsinszky, G. I. Csonka, O. A. Vydrov, G. E. Scuseria, L. A. Constantin, X. Zhou and K. Burke, Phys. Rev. Lett., 2008, 100, 136406.

25 G. Kresse and J. Furthmüller, Comput. Mater. Sci., 1996, 6, 15-50.

26 G. Kresse and J. Furthmüller, Phys. Rev. B: Condens. Matter Mater. Phys., 1996, 54, 11169-11186.

27 G. Kresse and D. Joubert, Phys. Rev. B: Condens. Matter Mater. Phys., 1999, 59, 1758-1775.

28 B. Himmetoglu, A. Floris, S. de Gironcoli and M. Cococcioni, Int. J. Quantum Chem., 2014, 114, 14-49.

29 M. Hellenbrandt, Cryst. Rev., 2004, 10, 17-22.

30 G. Henkelman and H. Jónsson, J. Chem. Phys., 2000, 113, 9978-9985.

31 G. Henkelman, B. P. Uberuaga and H. Jónsson, J. Chem. Phys., 2000, 113, 9901-9904.

32 N. G. Limas and T. A. Manz, RSC Adv., 2018, 8, 2678-2707. 33 T. A. Manz and N. G. Limas, $R S C A d v$., 2016, 6, 47771-47801. 34 N. G. Limas and T. A. Manz, RSC Adv., 2016, 6, 45727-45747. 35 M. Pavone, A. M. Ritzmann and E. A. Carter, Energy Environ. Sci., 2011, 4, 4933-4937.

36 V. Stevanovic, S. Lany, X. Zhang and A. Zunger, Phys. Rev. B: Condens. Matter Mater. Phys., 2012, 85, 115104.

37 J. Heyd, G. E. Scuseria and M. Ernzerhof, J. Chem. Phys., 2003, 118, 8207-8215.

38 R. Wahl, D. Vogtenhuber and G. Kresse, Phys. Rev. B: Condens. Matter Mater. Phys., 2008, 78, 104116.

39 K. K. Ghose, A. Bayon and A. J. Page, J. Phys. Chem. C, 2020, 124, 27055-27063.

40 J. J. Bown and A. J. Page, J. Mater. Chem. A, 2019, 7, 13029-13035. 